- Rubber dam is essential for effective isolation of the root canal and operating field from salivary bacteria, as well as protection of the airway.

- Only a very small number of rubber dam clamps are required for the efficient application of rubber dam, which must be supported by a well-trained dental nurse.

- Success in modern endodontic treatment may be dependent upon a well-designed access cavity to permit straight-line access to all the main root canals.

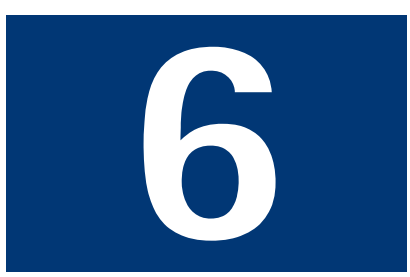

\title{
Endodontics: Part 6 \\ Rubber dam and access cavities
}

\author{
P. Carrotte ${ }^{1}$
}

NOW AVAILABLE AS A BDJ BOOK

Rubber dam is easy to apply once the basic components and principles are understood. An efficient and well-trained dental nurse will greatly facilitate the application procedure. Although preparation of the access cavity may be commenced before rubber dam is applied to enable anatomical landmarks to be followed, the rubber dam should be placed as soon as possible with adequate protection against contamination of the access. The access cavity reflects the shape of the pulp chamber, modified by the angle of instrument approach.

\section{ENDODONTICS}

1. The modern concept of root canal treatment

2. Diagnosis and treatment planning

3. Treatment of endodontic emergencies

4. Morphology of the root canal system

5. Basic instruments and materials for root canal treatment

6. Rubber dam and access cavities

7. Preparing the root canal

8. Filling the root canal system

9. Calcium hydroxide, root resorption, endo-perio lesions

10. Endodontic treatment for children

11. Surgical endodontics

12. Endodontic problems

${ }^{1 *}$ Clinical Lecturer, Department of Adult Dental Care, Glasgow Dental Hospital and School, 378 Sauchiehall Street, Glasgow G2 3JZ

${ }^{*}$ Correspondence to: Peter Carrotte

Email:p.carrotte@dental.gla.ac.uk

\section{Refereed Paper}

doi:10.1038/sj.bdj.4811799

(c) British Dental Journal 2004; 197

527-534

\section{RUBBER DAM}

The use of a rubber dam is almost mandatory in modern endodontic practice for three reasons.

Firstly, it provides an aseptic operating field, isolating the tooth from oral and salivary contamination. It cannot be stressed enough that contamination of the root canal with saliva introduces new microorganisms to the root canal which may prolong treatment and reduce prognosis.

Secondly, rubber dam facilitates the use of the strong medicaments necessary to clean the root canal system.

Finally, it protects the patient from the inhalation or ingestion of endodontic instruments, as shown in Figure 1.

Practitioners may also be advised to develop their rubber dam skills for another reason. Research has shown ${ }^{1}$ that rubber dam used during routine conservation procedures reduces aerosol contamination and cross-infection by up to $98.5 \%$.

Research has also shown that patients do not dislike the use of rubber dam, ${ }^{2}$ and the author has never had a single patient request its removal once the reasons for its use have been explained. It may be particularly helpful to explain to the patient that the rubber dam is necessary to isolate the operating area in exactly the same way as a surgical drape is essential for similar invasive medical procedures, where bacterial contamination may have a profound effect on the outcome. Indeed, refusal to accept a rubber dam may preclude the prescription of endodontic therapy. If an endodontic instrument is inhaled, a medicolegal allegation of negligence may be impossible to defend, even if the patient had appeared to accept the risk. A practitioner should never do anything to a patient which he or she knows to be wrong, and a patient may not sign away their rights in law.

With a little practice, an understanding of the basic principles, a well organised surgery and a well-trained dental nurse, a single tooth can be isolated in only a few seconds.

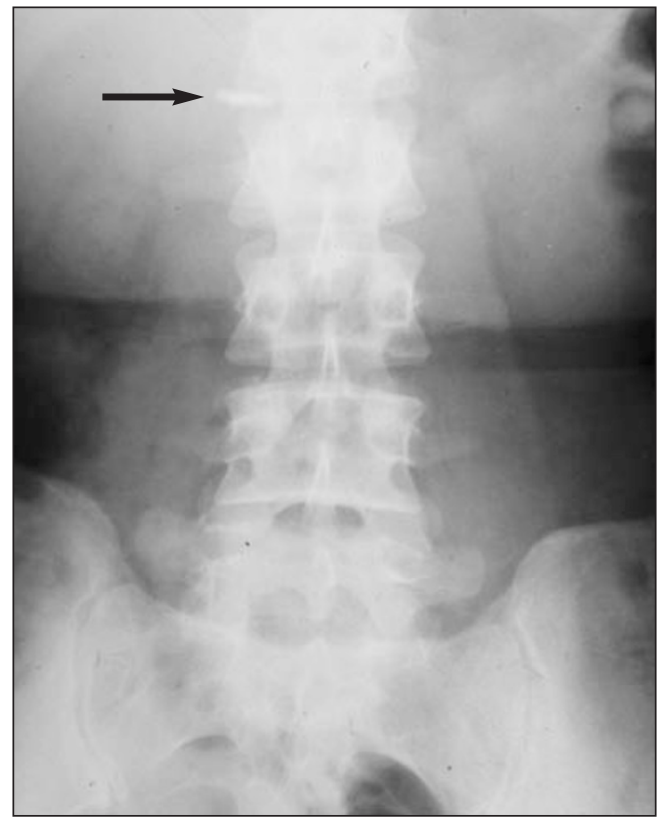

Fig. 1 An endodontic instrument has been inhaled due to a lack of airway protection. 


\section{PRACTICE}

Fig. 2 A modern single table rubber dam punch.

Fig. 4 The tips of the rubber dam forceps on the right have been modified for ease of use.

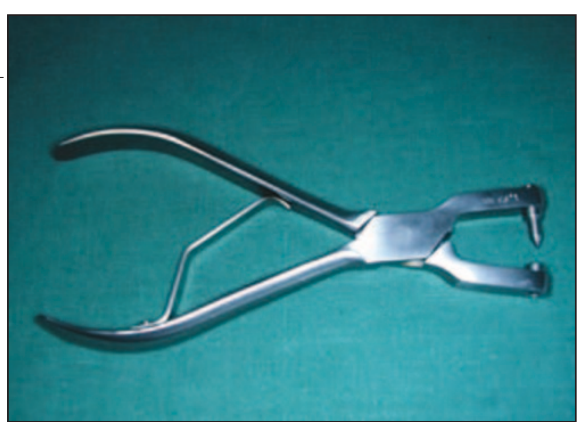

Fig. 3 For multiple isolation, the position of the holes to be punched may be marked by holding the rubber dam against the teeth.

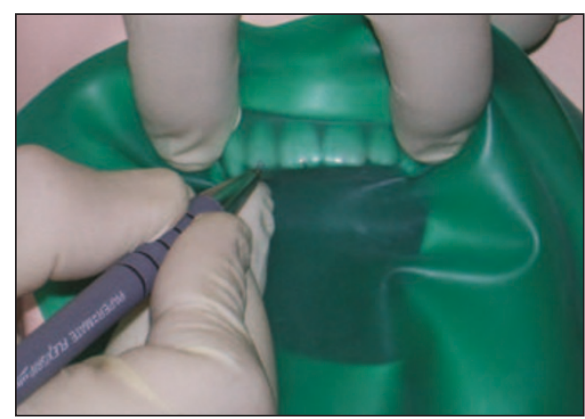

defect in the cut may cause the dam to split when stretched out. The new rubber dam punches are single table (Fig. 2) and should always cut a clean hole. If they do not, they should be returned to the supplier.

\section{Rubber dam stamp}

This is another piece of equipment now largely superseded. For single tooth isolation, a hole punched $2 \mathrm{~cm}$ diagonally from the middle of the sheet gives universal dam. The hole is simply orientated to the quadrant under treatment. For multiple isolation, it is preferable to hold the dam against the teeth to be isolated, and mark the centre of each tooth with a pen, as shown in Figure 3. The holes will then be punched in accordance with the patient's dentition and not with an arbitrary stamp.

\section{Rubber dam forceps}

The rubber dam forceps are used to carry the clamp to the tooth. The most frequently reported problem with rubber dam is that when the clamp has been expanded and placed on the tooth, the forceps are stuck in the clamp and cannot be removed! This is because the grooves in the tips of the forceps are too deep. These should be modified with a stone or sandpaper disc so that they just engage the clamp, but slide off easily. Most forceps have a second groove slightly distant from the tip, which may be used to remove the rubber dam clamp without re-engaging the holes (Figs 4 and 5). There are several designs of forceps, and they may be employed with either an over-hand or under-hand grip - experimentation will reveal the most comfortable.

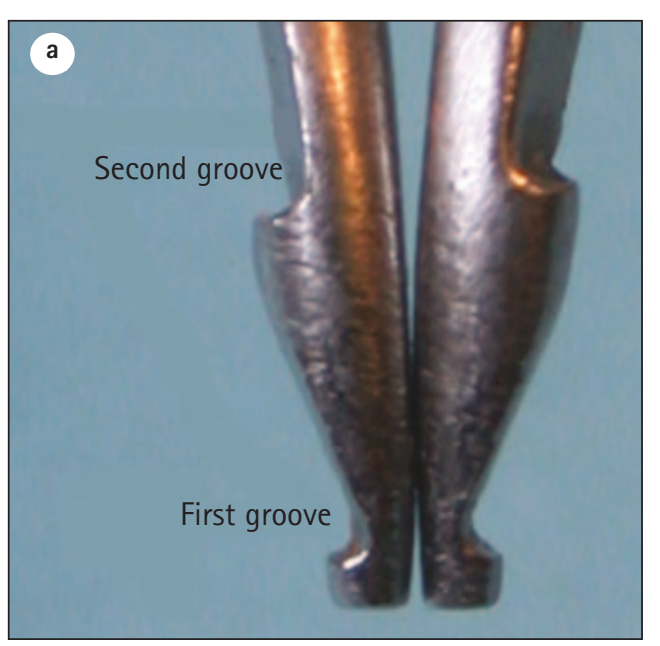

Fig. 5 a) The second groove for removal of the rubber dam clamp, as shown in b). 


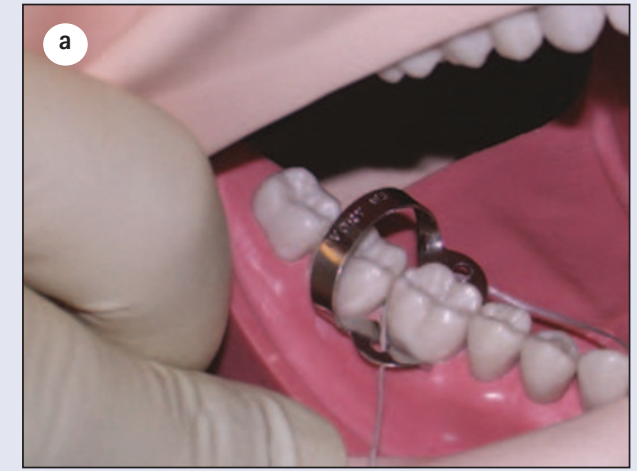

Fig. 6 Universal molar and premolar clamps,

demonstrated on a phantom head. a) A wingless molar clamp (W8a).

\section{Rubber dam clamps}

There is a vast range of shapes and sizes of rubber dam clamps, supposedly to suit every possible tooth and situation. In fact, this merely causes confusion, as an ill-fitting clamp may be quite unsatisfactory, and dislodge during treatment. When properly fitted, a clamp should have fourpoint contact with the tooth. If not, it will either rock back and forward or dislodge completely.

Clamps are described as being either 'active', where the jaws slope downwards and positively slide into cervical undercuts, or 'passive' when they tend to remain where placed. They may also be either winged or wingless, depending upon the chosen method of application.

The size 8A clamp is described by the manufacturers as a 'universal retentive molar clamp'.

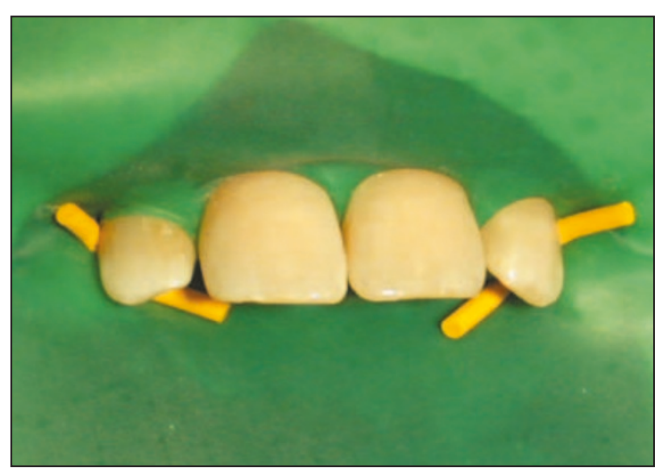

Fig. 7 Wedgets may be used in place of clamps for anterior teeth.

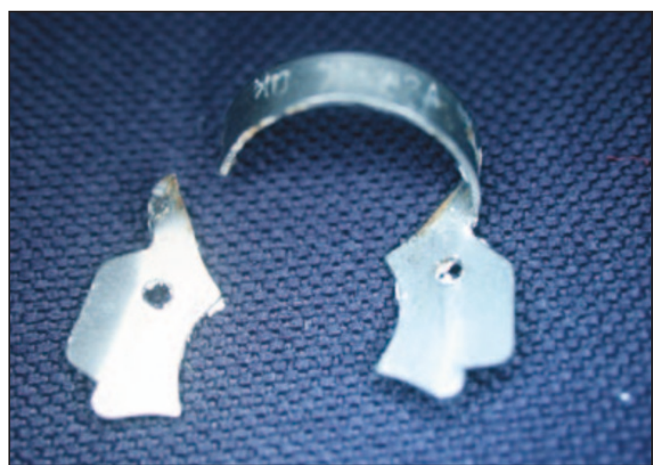

Fig. 8 Rubber dam clamps should always be protected with a length of dental floss in case they either fracture, as shown here, or are dropped in an unprotected mouth.

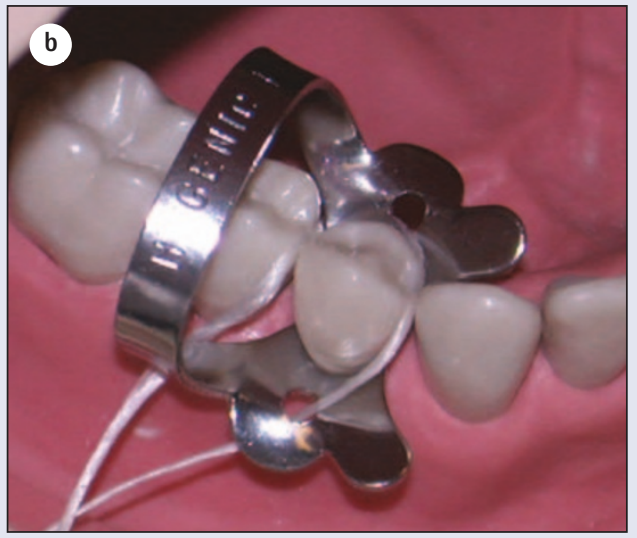

b) A winged premolar clamp (1)

It is an active clamp, and fits every molar tooth, even when these are quite broken down. The author would suggest therefore that all the other designs merely confuse the issue, and until the operator is very experienced only this clamp is used for all molar teeth. Likewise, the size 1 fits virtually all premolars (Fig. 6). If passive clamps are preferred size 0 or 00 are suitable for premolars, although they will not be as retentive. Rather than place aggressive clamps on anterior teeth, it is usually kinder to use interproximal wedges, either pieces of rubber dam or a commercial product such as 'Wedgets' (Fig. 7). It is often easier to isolate several anterior teeth, giving a clear operating field.

Occasionally, a clamp may be dropped in the patient's mouth, or may fracture across the bow as seen in Figure 8, during application. All rubber dam clamps should be protected with a length of dental floss, about $50 \mathrm{~cm}$, threaded through the holes on either side. It is not necessary for this to be wrapped around the clamp as was described in some early restorative textbooks. Indeed this should not be done since, once the clamp is in place above the rubber dam sheet and technically outside the mouth, the floss should be cut and withdrawn. If not it may act as a wick, drawing saliva into the operating field, or taking medicaments down into the mouth. Small voids around the dam may be sealed with a caulking agent such as Oraseal or Cavit (Fig. 9).
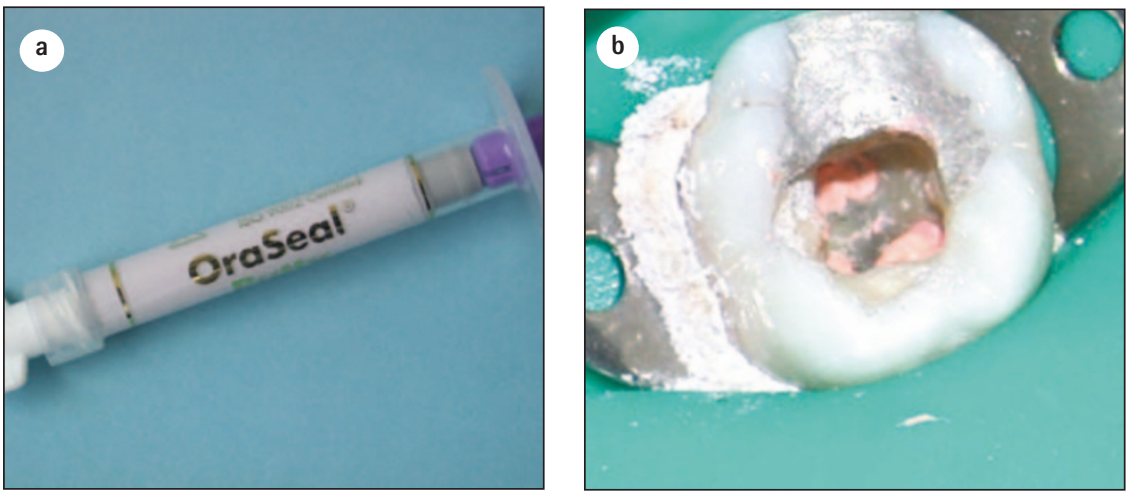

Fig. 9 a) A caulking agent which may be used to seal voids around the rubber dam that may allow salivary contamination, as shown in b). 


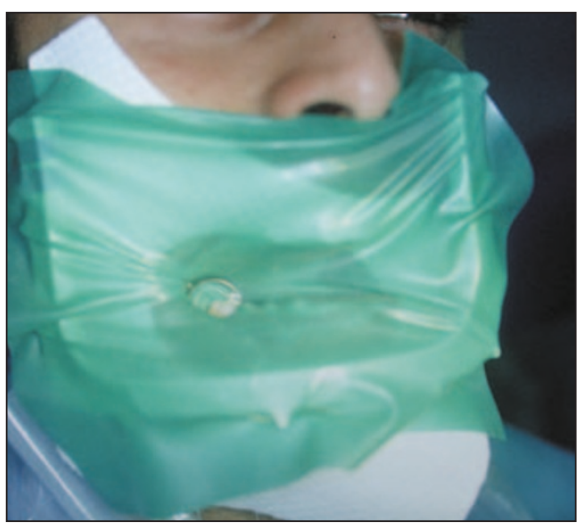

Fig. 10 The rubber dam frame may be easier to place beneath the rubber sheet.
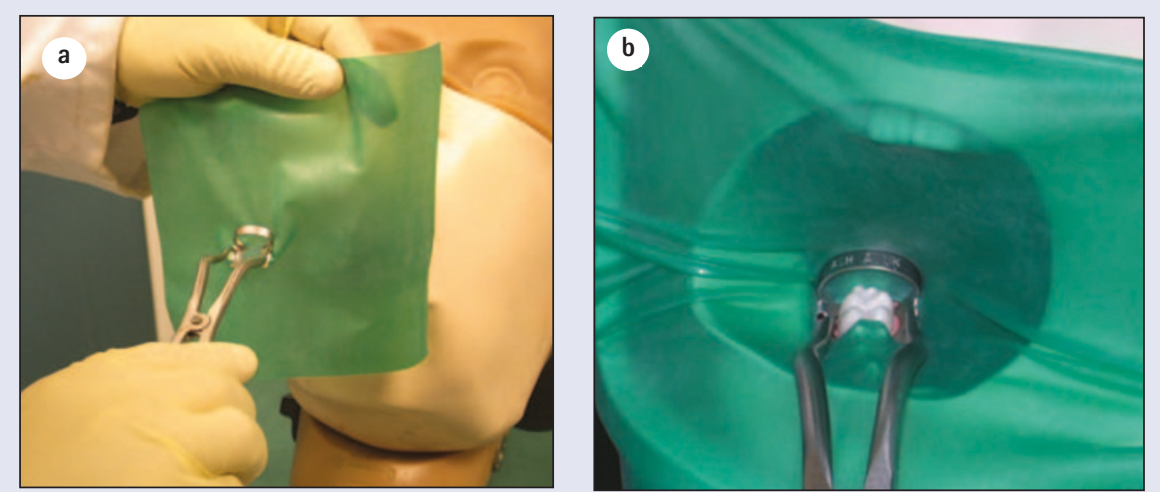

Fig. 11 The winged technique. The hole in the rubber sheet has been stretched over the wings of the clamp a), which is then fitted to the tooth b). The rubber is pushed off the wings, and the seal verified.

\section{The rubber dam frame}

The old Ash frame, with its 'butterfly' retainers, has largely been replaced by plastic or metal frames with sharp points or pins. It should be noted that the majority of these retentive points slope backwards, and the frames are designed to be placed under the rubber dam (Fig. 10). It seems to be much easier to place the frame beneath the dam and simply stretch the sheet over the points than the other way round. In addition, the tension in the sheet can be better controlled, particularly relevant when working in a situation where the clamp may be less retentive than normal.

For comfort, most patients appreciate a small piece of gauze placed between the rubber and their skin, and some practitioners place a prop (an old McKesson anaesthetic type) between the teeth on the non-working side to relieve the patient's muscle tension.

\section{APPLICATION TECHNIQUES}

A well-trained dental nurse and a well organised surgery, are essential for efficient application of the rubber dam. If a tray is prepared with ready punched sheets, and sterilised clamps already flossed, application can be performed in a matter of seconds.

There are three standard methods of application, described and illustrated here.

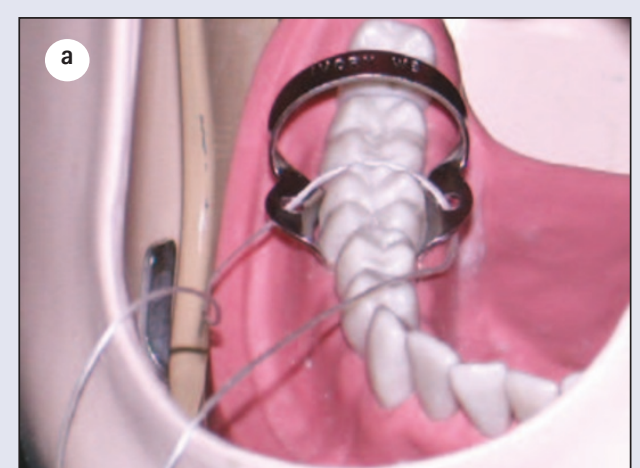

Fig. 12 The wingless technique. a) The flossed clamp has been placed on the tooth, and b) the rubber is stretched over the bow and pulled forward around the clamp.

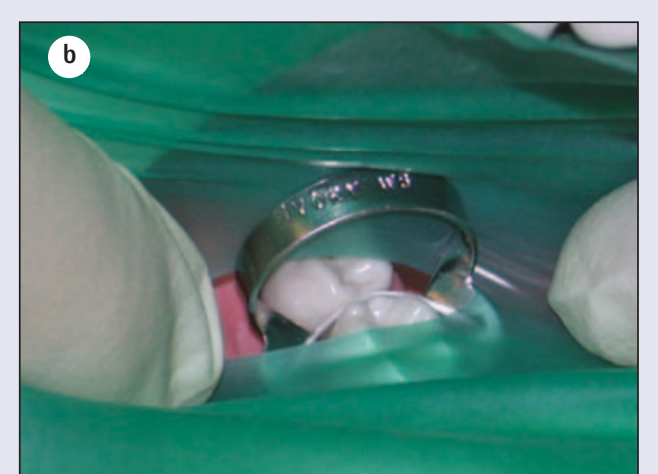

\section{Winged technique}

The appropriate winged clamp (8A for molars, 1 for premolars) is selected and flossed. The rubber dam is punched and aligned with the quadrant to be treated. The clamp is held in the forceps and retained with the ratchet. The hole in the rubber is stretched across the wings of the clamp, positioning the bow of the clamp towards the back of the arch. All this may be done by the dental nurse while the dentist is otherwise occupied, perhaps administering the local anaesthetic. The nurse then holds the top of the sheet to improve vision for the operator, who simply places the clamp onto the tooth to be treated (Fig. 11). A flat plastic instrument is then used to push the rubber off the wings, and the frame and gauze are applied. The floss may then be removed and the seal verified or adjusted as necessary.

\section{Wingless technique}

The appropriate wingless clamp (W8A for molars, W1 for premolars) is selected and flossed. The rubber dam is punched and aligned with the quadrant to be treated. The clamp, held in the forceps and retained with the ratchet, is placed securely on the tooth. One advantage of this method is that the opportunity now exists to verify the fit of the clamp before proceeding (Fig. 12). The rubber dam is now held in both hands, and the index fingers used to stretch out the punched hole, which is slipped over the bow 


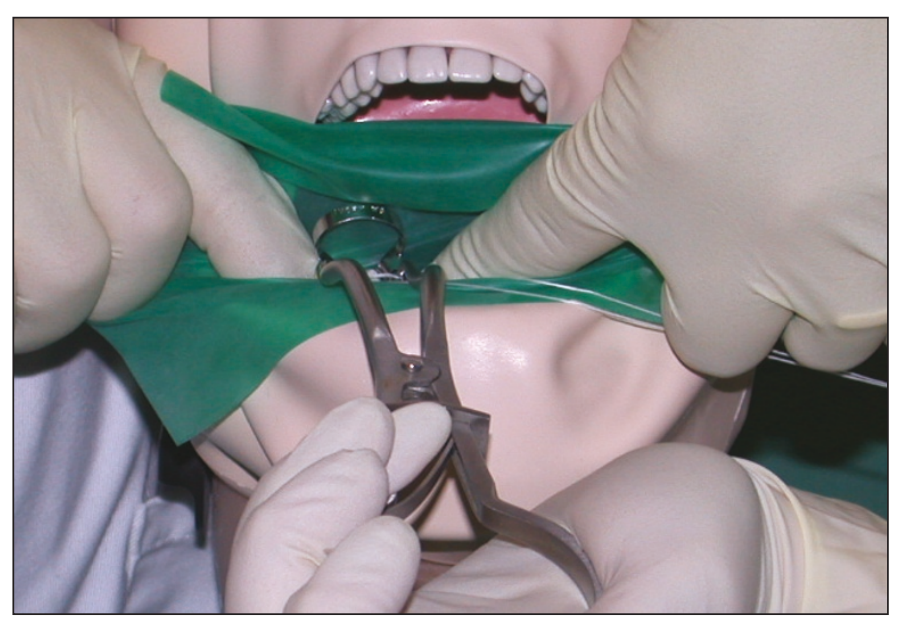

Fig. 13 The rubber first method. The operator is holding the sheet over the tooth whilst the assistant places the clamp to secure it in place.

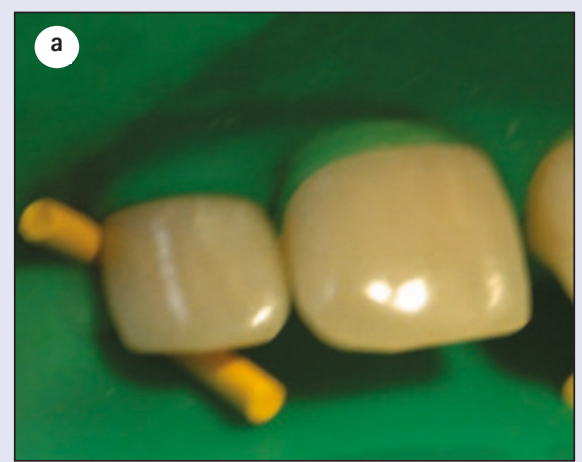

Fig. 14 a) The rubber dam is lying on the tooth surface and may allow leakage. It should be everted into the gingival crevice by

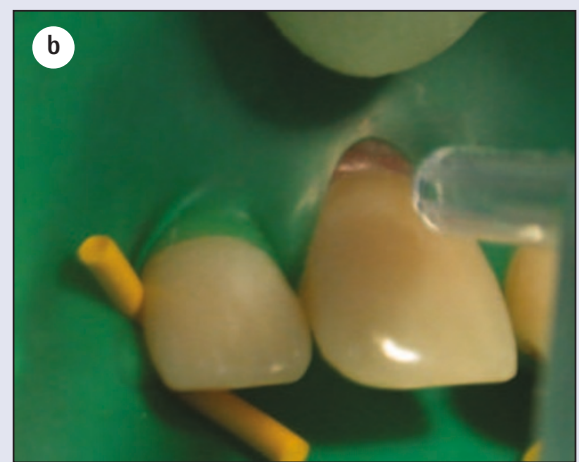

b) stretching the rubber away from the tooth and drying the mucosa with a stream of cold air, before

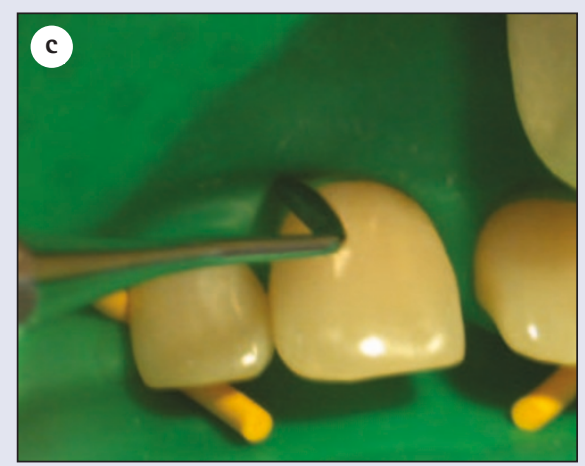

c) using a flat plastic instrument to tuck the rubber into the crevice. of the clamp and pulled forward and down onto the tooth. Again, the nurse may hold the top of the sheet to improve vision for the operator. The frame and gauze are applied, the floss removed and the seal verified or adjusted as necessary.

\section{Rubber first}

The third method taught in some centres involves the dental nurse to a greater extent. The dentist stretches out the rubber and places the hole over the tooth in question, holding it down on each side with light finger pressure. At the same time the dental nurse picks up the flossed clamp in the forceps and places it over the tooth, retaining the dam in place (Fig. 13). Once again, the frame and gauze are applied, the floss removed and the seal verified or adjusted as necessary.

\section{Anterior teeth}

As stated previously, in a relatively intact arch it is easier to isolate several anterior teeth. Dental floss should be used first to verify that the contacts are clear and that the rubber dam will pass through. The rubber sheet is held against the teeth and the centre point marked of those teeth to be isolated. Holes are punched at these points, and the rubber dam is then applied to the teeth. Taking a leading edge of rubber through the contact - 'knifing through' - makes application relatively simple, or dental floss may be used to draw the rubber through a difficult contact. Once in place the selected wedges are applied.

\section{Everting the margins}

If the rubber dam is lying on the tooth surface it may allow leakage of saliva, a problem particularly when the dam has been applied during adhesive restorative procedures, but also with endodontics. The margin should be everted into the gingival crevice as shown in Figure 14. The operator stretches the rubber away from the tooth whilst the assistant directs a stream of cold air from the triple syringe onto the mucosa. With the use of a flat plastic instrument the margin of the rubber dam may be tucked into the gingival crevice, providing a tight seal.

Alternatively, some operators apply floss ligatures, as shown in Figure 15, using a flat plastic

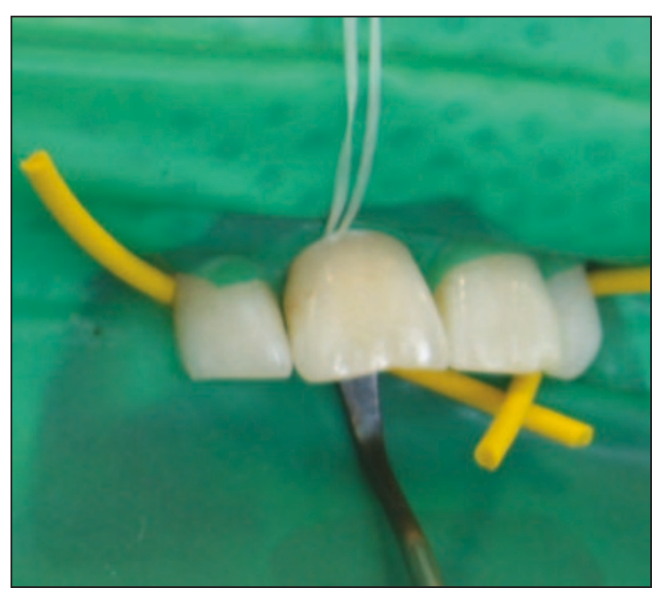

Fig. 15 Alternatively, floss ligatures may be used to hold the rubber dam in the gingival crevice. Ligatures will be applied to each tooth under treatment. 

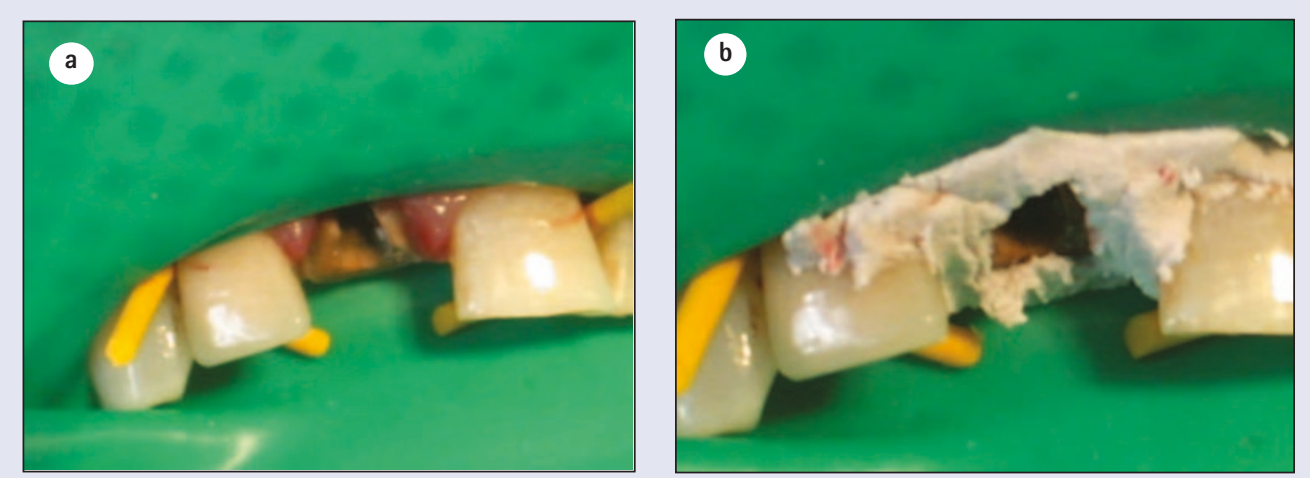

Fig. 16 a) A slot has been cut in the rubber dam to enable this root to be isolated. b) However, it is essential that a caulking material is applied to prevent salivary contamination.

to push the floss above the cingulum, and tying a knot securely on the labial aspect of the tooth.

\section{DIFFICULT SITUATIONS}

There are a few situations where the application of rubber dam may present difficulties, although the cautious clinician may consider whether root canal treatment is then either appropriate, or may be severely compromised.

\section{The broken down tooth}

The broken down tooth may be tackled in a variety of ways. Many molar teeth with large deficiencies may have rubber dam applied, providing the right clamp is used; the author recommends a W8a (see Fig. 2). With an appropriate length of floss as described earlier, the clamp is placed directly on to the tooth, so that there is a four-point contact between the jaws of clamp and the root. Once in position, the clamp is checked for stability by pressing on the bow. If firm, the rubber dam may be stretched over the clamp using the wingless technique described.

It is also feasible to build up the tooth before commencing root canal treatment using glass ionomer (for example, Vitremer). Alternatively, an orthodontic band may be cemented around the tooth. On occasion, a clamp may be fitted on to a broken down tooth, but only if the gingival tissue encroaching on to the margin is first removed with electrosurgery or a surgical blade.

As part of the restorative treatment, periodontal crown lengthening, or orthodontic extrusion, may be indicated. These procedures should be carried out prior to commencing the root canal treatment.

\section{Bridges}

Bridges do not present a problem with the application of rubber dam. A suitable winged clamp is fitted onto the abutment tooth and the dam stretched over the clamp. If there are any small gaps, these can be sealed with a caulking material such as Oraseal or Cavit.

When root treating teeth acting as bridge abutments a careful check should always be made that the bridge is not loose. If a bridge has become debonded it must be removed. This should be carried out before any attempt is made to root-treat one of the abutment teeth.

\section{Split dam}

Occasionally, a broken down tooth may be isolated using a slit cut between the holes made for the two adjacent teeth, as shown in Figure 16. It is essential that the caulking material illustrated in Figure 9 is applied to prevent leakage and contamination.

Finally, if an operator decides to proceed with root canal treatment without the use of rubber dam, each hand file must be protected with either floss tied around the handle or an appropriate safety device. The excess saliva must be controlled with cotton wool rolls and aspiration, and great care must be taken with medicaments. It would be advisable to inform the patient of the risks involved, and the reduced prognosis for the treatment if salivary contamination of the root canal occurs.

\section{ACCESS}

\section{Access cavity preparation}

There is an old cliché that 'Access is Success'. Unlike other aspects of dentistry, root canal treatment is carried out with little visual guidance; therefore, the difficulties that are likely to be encountered need to be considered. An assessment of the following features can be made after visual examination of the tooth, and study of a pre-operative periapical radiograph taken with a paralleling technique:

- The external morphology of the tooth.

- The architecture of the tooth's root canal system.

- The number of canals present.

- The length, direction and degree of curvature of each canal.

- Any branching or division of the main canals.

- The relationship of the canal orifice(s) to the pulp chamber and to the external surface of the tooth.

- The presence and location of any lateral canals.

- The position and size of the pulp chamber and its distance from the occlusal surface.

- Any related pathology.

Before commencement of root canal treatment, the tooth must be prepared as follows: 


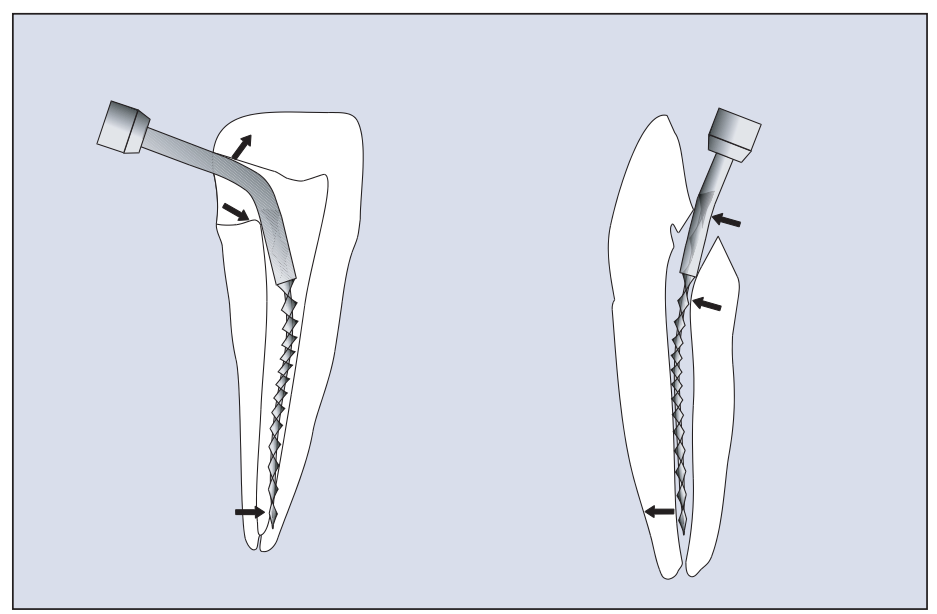

Fig. 17 This diagram illustrates the importance of straight line access and correctly designed access cavities. If root canal treatment were to be carried out through a Class III cavity, as shown on the left, the file would be deflected and the canal would be transported. However, if the access cavity is cut incorrectly in the palatal surface, not giving straight line access, the same deflection and damage will occur.

- All caries and any defective restorations should be removed and made good. The tooth should be protected against fracture during treatment.

- The tooth should be capable of isolation.

- The periodontal status should be sound, or capable of resolution.

It may be prudent to commence access cavity preparation before isolating the tooth with rubber dam in order that the anatomical landmarks, tooth inclination and other helpful features are not lost. It is, of course, crucial that the root canal does not become contaminated during either access preparation or canal instrumentation, and the tooth should be isolated in an aseptic field as soon as possible.

If there is a danger of fracture of the coronal tooth structure, the cuspal height should be reduced to prevent this. If the loss of coronal tissue is extensive, there may be a need to provisionally restore the tooth with a temporary crown, copper ring or an orthodontic band. It is, however, not always necessary to restore the tooth before carrying out endodontic procedures. Provided the tooth will anchor a rubber dam, the canals can be isolated from the oral cavity and a temporary seal can be placed over the canals, this will be sufficient.

The objectives of access cavity preparation are to:

- Remove the entire roof of the pulp chamber so that the pulp chamber can be debrided.

- Enable the root canals to be located and instrumented by providing direct straight line access to the apical third of the root canals, as illustrated in Figure 6.17. Note that the initial access cavity may have to be modified during treatment to achieve this.

- Enable a temporary seal to be placed securely in order to withstand any displacing forces.

- Conserve as much sound tooth tissue as possible and as is consistent with treatment objectives.

The subsequent restoration of the tooth should always be considered first. If the tooth is not heavily restored then only the amount of coronal tissue sufficient for the successful completion of the root canal treatment should be removed. However, if the tooth is already compromised and will require some form of cuspal coverage restoration, an onlay or a crown, then it may be practical to reduce the cusp height, particularly mesiobuccally in molars, to enable better visualisation of the pulp chamber. If access to the back of the mouth is difficult, it is again reasonable to consider reducing the marginal ridge of the tooth concerned to achieve this (Fig. 18), or perhaps gain access through the mesiobuccal wall. Unless the root treatment is successful, any further restoration to the tooth will be put at risk.

Before beginning the access cavity preparation, it is wise to check the depth of the preparation by aligning the bur and handpiece against the radiograph, in order to note the position and depth of the roof of the pulp chamber in relation to the length of the bur in the handpiece (Fig. 19). Particular note should be made of the position of the largest pulp horn.

The stages of access cavity preparation may be summarised as follows:

1. The initial entry is made with a tungsten carbide or diamond bur in a turbine handpiece and the outline form completed as required. The bur is advanced towards the pulp horns until the roof of the pulp chamber is just penetrated. (Note particularly that in a molar tooth the bur approaches the tooth from the mesial and from the buccal. Thus the access

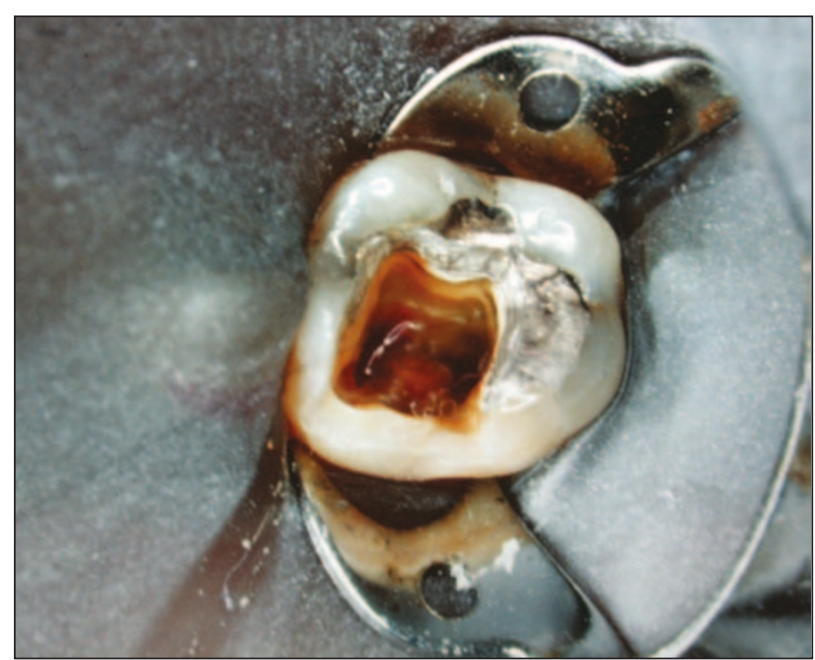

Fig. 18 Reducing the mesial marginal ridge may be necessary to permit clear visualisation of the pulp chamber. 
Fig. 19 The pre-operative radiograph should be examined carefully for suitable landmarks before commencing the access cavity. Here, the depth of penetration of the bur is being estimated.

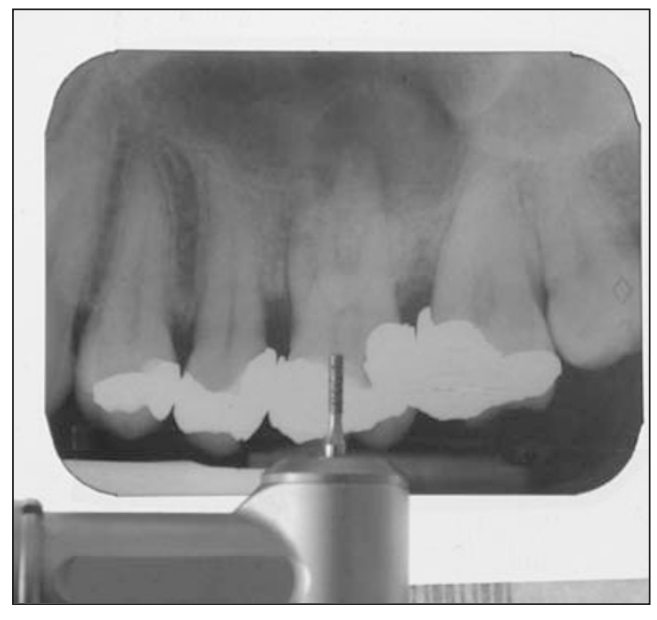

cavity is cut in the mesiobuccal segment of the occlusal surface.)

2. At this point, the rubber dam should be applied if it is not already in place.

3. The removal of the entire roof of the pulp chamber, and the tapering of the walls, is now carried out with a safe-tipped endodontic access bur, as described in Part 5. (Stages 1 and 3 are illustrated in the diagrams in Fig. 20.)

4. The walls of the pulp chamber may now be gently flared out towards the occlusal surface. The end result should be a gentle funnelshape, with the larger diameter at the occlusal surface. The safe tip of the bur will be felt passively following the contours of the floor of the pulp chamber.

5. Any remaining pulp tissue and debris is cleared with an excavator from the floor of the pulp chamber and the canal orifices.

6. The access cavity should be flushed with a solution of sodium hypochlorite to remove any residual debris.

7. The canal orifices may be located with a DG 16 endodontic probe. Any alteration to the access cavity outline form may now be undertaken to ensure a direct line of approach to the canal orifices. Any sclerotic or secondary dentine surrounding the canal orifices may be removed with a CT4 tip in a piezo-electronic ultrasonic machine.

8. Once the canal orifices have been identified, the preparation of the coronal part of the root canals should be commenced. Depending upon the operator's preferred technique, either Gates-Glidden burs or nickel-titanium orifice shapers, should be employed. Copious irrigation is necessary, together with the use of a canal lubricant containing EDTA. These techniques are described in Part 7.

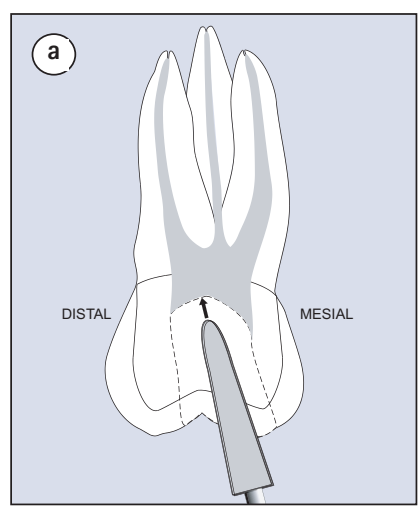

Fig. 20 These diagrams illustrate how, in a molar tooth, the bur approaches the pulp chamber from the mesial (a) and from the buccal (b). When the roof of the pulp chamber has been penetrated, a safe-tipped bur (c) should be used to remove the roof of the pulp chamber and shape the

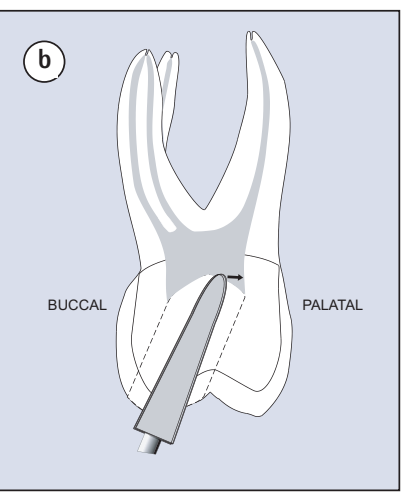
walls without damaging the floor and canal orifices.

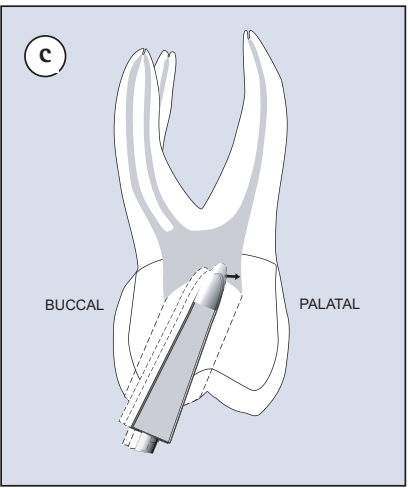

9. An access cavity must be seen as dynamic, with modifications being made as treatment progresses to permit the straightest possible access of instruments.

10. It can only be repeated that access is success. If the access cavity is not large enough to permit easy and thorough shaping and cleaning of the entire root canal system, the root canal treatment may be compromised and the tooth lost.

1. Marshall K. Dental workspace contamination and the role of rubber dam. CPD Dentistry 2001; 2: 48-50.

2. Gergely E J. Rubber dam acceptance. $B D J 1989 ; 167$ : 249-252. 